\title{
Higher preconceptional maternal body mass index is associated with faster early preimplantation embryonic development: the Rotterdam periconception cohort
}

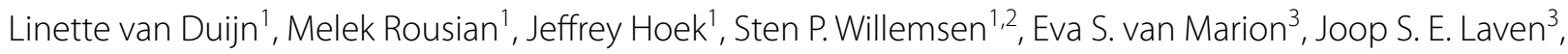
Esther B. Baart ${ }^{3,4+}$ and Régine P. M. Steegers-Theunissen ${ }^{1 *+}$

\begin{abstract}
Background: Overweight and obesity affect millions of people globally, which has also serious implications for reproduction. For example, treatment outcomes after in vitro fertilisation (IVF) are worse in women with a high body mass index (BMI). However, the impact of maternal BMI on embryo quality is inconclusive. Our main aim is to study associations between preconceptional maternal BMI and morphokinetic parameters of preimplantation embryos and predicted implantation potential. In addition, associations with clinical IVF outcomes are investigated.

Methods: From a tertiary hospital, 268 women undergoing IVF or IVF with intracytoplasmic sperm injection (ICSI) were included; 143 normal weight, 79 overweight and 46 obese women. The embryos of these women were cultured in the EmbryoScope, a time-lapse incubator. The morphokinetic parameters of preimplantation embryos and predicted implantation potential, assessed by the KIDScore algorithm were longitudinally evaluated as primary and secondary outcomes, respectively. The tertiary outcomes included clinical outcomes, i.e., fertilization, implantation and live birth rate.
\end{abstract}

Results: After adjustment for patient- and treatment-related factors, we demonstrated in 938 embryos that maternal BMI is negatively associated with the moment of pronuclear appearance $\left(\beta_{\mathrm{tPNa}}-0.070 \mathrm{~h}(95 \% \mathrm{Cl}-0.139,-0.001)\right.$, $p=0.048)$, pronuclear fading $\left(\beta_{\mathrm{tPNf}}-0.091 \mathrm{~h}(95 \% \mathrm{Cl}-0.180,-0.003), p=0.043\right.$ and the first cell cleavage $\left(\beta_{\mathrm{t} 2}-0.111 \mathrm{~h}\right.$ $(95 \% \mathrm{Cl}-0.205,-0.016), p=0.022)$. Maternal BMI was not significantly associated with the KIDScore and tertiary clinical treatment outcomes. In embryos from couples with female or combined factor subfertility, the impact of maternal BMI was even larger ( $\left.\beta_{\mathrm{tPNf}}-0.170 \mathrm{~h}(95 \% \mathrm{Cl}-0.293,-0.047), p=0.007 ; \beta_{\mathrm{t} 2}-0.199 \mathrm{~h}(95 \% \mathrm{Cl}-0.330,-0.067), p=0.003\right)$. Additionally, a detrimental impact of BMI per point increase was observed on the KIDScore ( $\beta-0.073$ (se 0.028$), p=0.010$ ).

Conclusions: Higher maternal BMI is associated with faster early preimplantation development. In couples with female or combined factor subfertility, a higher BMI is associated with a lower implantation potential as predicted by the KIDScore. Likely due to power issues, we did not observe an impact on clinical treatment outcomes. However, an

\footnotetext{
*Correspondence: r.steegers@erasmusmc.nl

${ }^{\dagger}$ Esther B. Baart and Régine P.M. Steegers-Theunissen contributed equally to this work.

1 Department of Obstetrics and Gynecology, Erasmus MC, University

Medical Center, Dr. Molewaterplein 40, Rotterdam 3015 GD, The Netherlands

Full list of author information is available at the end of the article
} permits use, sharing, adaptation, distribution and reproduction in any medium or format, as long as you give appropriate credit to the original author(s) and the source, provide a link to the Creative Commons licence, and indicate if changes were made. The images or other third party material in this article are included in the article's Creative Commons licence, unless indicated otherwise in a credit line to the material. If material is not included in the article's Creative Commons licence and your intended use is not permitted by statutory regulation or exceeds the permitted use, you will need to obtain permission directly from the copyright holder. To view a copy of this licence, visit http://creativecommons.org/licenses/by/4.0/. The Creative Commons Public Domain Dedication waiver (http://creativeco mmons.org/publicdomain/zero/1.0/) applies to the data made available in this article, unless otherwise stated in a credit line to the data. 
effect of faster preimplantation development on post-implantation development is conceivable, especially since the impact of maternal BMI on pregnancy outcomes has been widely demonstrated.

Keywords: Body mass index, Time-lapse, Morphokinetics, Embryo development, Preimplantation

\section{Introduction}

Overweight and obesity affect millions of people of all ages, genders, ethnicities and income levels [1]. Although the pathophysiology of adiposity is highly complex and multifactorial, it is fundamentally caused by a positive energy imbalance and influenced by genetic and numerous environmental factors [2, 3]. Surplus energy is stored as fat, which leads to a disruption of numerous physiological processes on endocrine, immune and vascular levels [4]. This explains why an elevated body mass index (BMI) is associated with various non-communicable diseases, such as diabetes type 2 and cancer $[5,6]$.

Obesity and overweight not only increase the risk of non-communicable diseases, but also can impact reproduction [7]. As almost half of the women in the reproductive period are overweight or obese, this has serious consequences. Adiposity affects metabolic and endocrine processes involved in fertility, which leads to an increased risk of miscarriages, reduced conception rate and anovulation [8-10]. Therefore, overweight and obesity are likely overrepresented in women receiving fertility treatment, such as in vitro fertilization (IVF) [11].

Outcomes after IVF treatment are poorer in women with a high BMI compared to normal weight women [12-14]. The mechanisms by which adiposity affects reproduction are not yet fully understood. It is suggested that obesity interferes with biological processes and pathways at endocrine, follicular, uterine and embryonic levels [15-18]. For example, obesity increases follicular fluid concentrations of lipids, metabolites and inflammatory markers, and impacts gene expression of cumulus cells, which can impair oocyte development $[19,20]$. Interestingly, the impact of maternal BMI on embryo quality is inconclusive [18, 21-23].

Since three decades, preimplantation embryo development can be closely observed with time-lapse imaging [24]. This technique is increasingly used to study associations between embryo development and implantation, and to improve embryo selection by algorithms such as the KIDScore [25]. Prospective randomized trials report conflicting results on the improvement of success rates after embryo selection based on timelapse parameters, and also indicate that these parameters are subject to patient-related factors [26, 27].
Moreover, previous studies investigating the impact of BMI on these parameters report conflicting results and are exclusively performed in cycles with intracytoplasmic sperm injection (ICSI) [28, 29].

From this background, the main aim of this study is to investigate the hypothesis that a high maternal BMI is detrimentally associated with preimplantation embryo quality, as assessed by developmental time-lapse parameters and predicted implantation potential (KIDScore). In addition, we also investigated associations with clinical treatment outcomes after IVF/ICSI treatment.

\section{Methods \\ Study design}

The data used for this study was collected between May 2017 and December 2019 as part of the Virtual Embryoscope study. This is an ongoing prospective sub-study of the Rotterdam Periconception Cohort, an observational open prospective tertiary hospital-based cohort, embedded in the outpatient clinic of the Department of Obstetrics and Gynaecology of the Erasmus MC, University Medical Center, Rotterdam, The Netherlands [30]. The Rotterdam Periconception Cohort focuses on periconceptional influences on reproductive success and adverse pregnancy outcomes and health of the offspring up to 1 year of age. For this study, subfertile couples with an indication for IVF/ICSI treatment, aged 18 years or older with adequate understanding of the Dutch language were eligible for participation.

For this study, couples were excluded if: 1) no fertilization occurred and no embryos were available $(n=14)$; 2 ) the embryos were not cultured in the EmbryoScope $(n=35)$; 3) IVF/ICSI treatment was performed with donated or vitrified oocytes $(n=8) ; 4)$ treatment was performed after $>1$ year following inclusion in the study $(n=5)$ or 5) ICSI was performed with testicular extracted sperm (Fig. 1).

\section{In vitro fertilization procedures and time-lapse imaging}

Ovarian stimulation, oocyte retrieval and IVF/ICSI procedures were performed as previously described [31, 32]. Women underwent ovarian stimulation with either recombinant follicle stimulating hormone $(\mathrm{rFSH})$ or urinary $\mathrm{FSH}$, with gonadotrophin-releasing hormone $(\mathrm{GnRH})$ agonist or GnRH-antagonist co-treatment. Ovarian stimulation protocols were standardized; the distribution of $\mathrm{GnRH}$-agonist or -antagonist protocol 


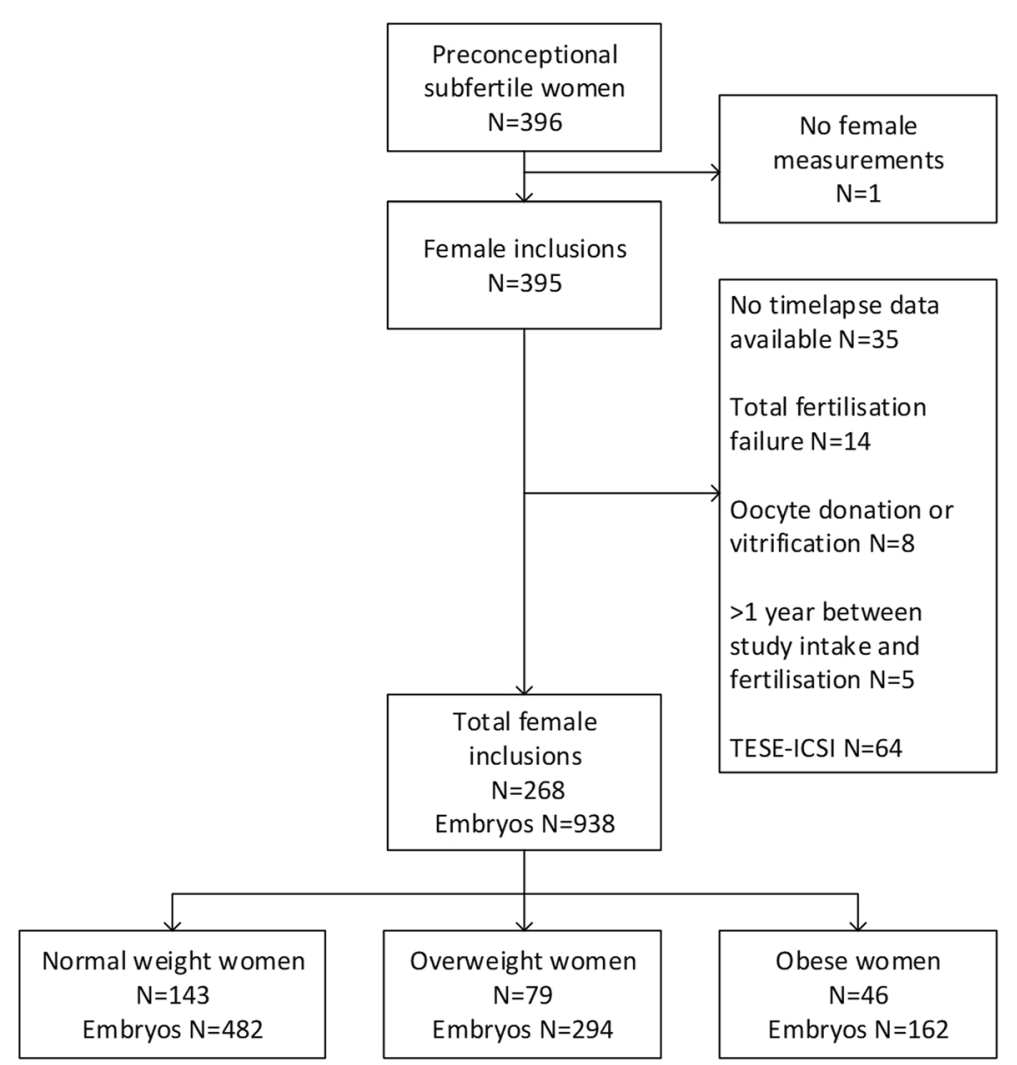

Fig. 1 Flowchart of the VIRTUAL EmbryoScope study population embedded in the Rotterdam Periconception Cohort. Abbreviations: TESE, testicular sperm extraction. ICSI, intracytoplasmic sperm injection

reflects policy changes over time and not patient selection. FSH dosage was based on maternal age, BMI, antral follicle count, anti-müllarian hormone (AMH) level and prior response to gonadotrophins (if applicable). Final follicular maturation was triggered with human chorionic gonadotrophin (hCG) or a $\mathrm{GnRH}$-agonist. Oocytes were collected $35 \mathrm{~h}$ later and cultured in SAGE human tubal fluid medium (HTF, CooperSurgical, Trumbull, CT, USA); supplemented with $5 \%$ human serum albumin (CooperSurgical) under an oil overlay (CooperSurgical).

After insemination, IVF oocytes were cultured overnight in drops of $100 \mu \mathrm{l}$ HTF medium in universal GPS dishes (CooperSurgical) under oil. The next morning, only fertilized dipronucleate oocytes were transferred to an EmbryoSlide (Vitrolife, Goteborg, Sweden) for culture in the EmbryoScope ${ }^{\mathrm{TM}}$ time-lapse incubator (Vitrolife). ICSI oocytes were denuded and transferred to an EmbryoSlide directly after sperm injection. Injected oocytes or embryos were cultured individually in $25 \mu \mathrm{l}$ of SAGE 1-Step medium (Cooper Surgical) under $1.4 \mathrm{ml}$ oil. Culture in the EmbryoScope ${ }^{\mathrm{TM}}$ was conform conventional culture, performed using customized settings, with a temperature setting of $36.8^{\circ} \mathrm{C}$ and in an atmosphere containing $7 \% \mathrm{O}_{2}$ and $4.5 \% \mathrm{CO}_{2}$. This atmosphere was validated to result in a $\mathrm{pH}$ of the SAGE 1-Step culture medium of 7.2-7.3.

Images were automatically recorded every $10 \mathrm{~min}$ after exposure to a single red LED $(635 \mathrm{~nm},<0.1 \mathrm{~s}$ per image) with a monochrome CCD camera. On day 3 after fertilization, embryo evaluation and selection for embryo transfer (ET) was based on conventional morphology, i.e. number of blastomeres, fragmentation, size equality, and signs of early compaction, without the support of timelapse information. Selection of embryos for cryopreservation was performed on day 4 , based on the degree of compaction and fragmentation.

Two weeks after ET, implantation was biochemically confirmed by a positive $\beta$-hCG test. Pregnancy was confirmed by ultrasound at 7 and 12 weeks of gestation by the presence of a gestational sac and fetal heartbeat.

\section{Study parameters}

Participants completed a preconceptional questionnaire covering demographic and lifestyle details. A researcher verified all data at study entry and measured anthropometrics. Subfertility diagnoses were retrieved from 
medical records and, when applicable, stratified according to the WHO classification of anovulation [33].

Time-lapse parameters were annotated manually according to the definitions of the ESHRE consensus for dynamic monitoring of human preimplantation development [34]. All freshly transferred and cryopreserved embryos were annotated for the following morphokinetic parameters: pronuclear appearance $(\mathrm{tPNa})$, pronuclear fading (tPNf), t2, t3, t4, t5, t6, t7 and t8. tPNa was defined as the first frame in which both pronuclei had appeared, and tPNf as the first frame in which both had faded. Timing of reaching the 2-, 3-, 4-, 5-, 6-, 7-, and 8-cell stage was defined as $t 2, t 3, t 4, t 5, t 6, t 7$ and $t 8$, respectively. Some of these parameters were used to assign each embryo a KIDScore (Supplemental table 1). This is a generally applicable embryo deselection tool based on 6 parameters, of which the lowest score $(=1)$ corresponds with a chance of implantation of $5 \%$, whereas the highest score $(=5)$ corresponds with a $36 \%$ chance of implantation [25]. Validation of the KIDScore in our clinic showed that KIDScore 1 embryos implant in 23\% of cases, increasing to $52 \%$ for KIDScore 5 embryos after SET. Internal validation of inter-observer reproducibility demonstrated extremely close agreement for the timings of tPNf until t5 (intraclass correlation coefficient (ICC) $>0.95$ ). A moderate agreement was found for $t 6, t 7$ and $\mathrm{t} 8$ (ICC 0.23-0.40).

Clinical treatment outcomes were retrieved from medical records. Pre-transfer clinical treatment outcomes included: fertilization rate, which was calculated by dividing the number of fertilized oocytes by the number of metaphase II oocytes retrieved and embryo usage rate, which was calculated by dividing the number of usable embryos, i.e. all freshly transferred and cryopreserved, by the number of fertilized oocytes. Post-transfer clinical treatment outcomes included cumulative pregnancy rate, which was defined as an ongoing pregnancy resulting from either fresh ET or frozen-thawed ET from the studied treatment cycle within a 2 year follow-up period.

\section{Statistical analyses}

Based on initial reports in human embryos, preimplantation embryos from obese women are expected to be developmentally delayed [35]. Based on culture of the first 900 embryos in our EmbryoScope ${ }^{\mathrm{TM}}$, average time needed from pronuclear disappearance to the 4-cell stage was $16.7 \mathrm{~h}(\mathrm{SD} \pm 5)$. The intra-cluster correlation coefficient (ICC) was 0.16 . To show a delay of at least $2 \mathrm{~h}(0.4 \mathrm{SD})$ in reaching the 4-cell stage, while correcting for statistical clustering of embryos derived from the same patient ( $1+([$ average no. of embryos/ patient $]-1) *$ ICC $=1+4 * 0.1591=1.64$ ), we needed $100 *$ $1.64=164$ embryos in each group to achieve an $80 \%$ power to detect this $2 \mathrm{~h}$ difference at $\alpha=0.05$.

Continuous baseline data were compared between women with normal weight, overweight and obesity, using Kruskall-Wallis tests for continuous data and chisquare tests for categorical data.

Analyses of morphokinetic parameters were performed on transferred and cryopreserved embryos. Since couples often have multiple embryos and embryos from a couple are likely to exhibit comparable developmental patterns, linear regression analyses are not appropriate. Therefore, we applied linear mixed models with time-lapse parameters as response variables and BMI as independent variable.

Proportional odds models were used to study the association between maternal BMI and the KIDScore, using the ordinal package in $\mathrm{R}$ (Rune Haubo B Christensen). This model is for ordinal outcomes like the KIDScore, with patient-specific intercepts to account for the correlation between sibling embryos.

Associations between BMI and continuous treatment outcomes, such as fertilization rate, were analyzed using linear regression. For associations between BMI and dichotomous outcomes, such as a positive $\beta$-hCGtest, logistic regression was applied.

All associations were studied with maternal BMI as a continuous variable. Two models were constructed for analyses on morphokinetic parameters and the KIDScore; a crude model without adjustments and the adjusted model with adjustments for maternal age, fertilization method, type of ovarian stimulation and paternal BMI and age. Analyses on treatment outcomes were adjusted for maternal age and type of ovarian stimulation. Post-hoc analyses were performed with maternal BMI divided into categories of normal weight $\left(\mathrm{BMI} \leq 25 \mathrm{~kg} / \mathrm{m}^{2}\right)$, overweight $(\mathrm{BMI}>25 \mathrm{~kg} /$ $\mathrm{m}^{2}$ ) and obese (BMI $\left.>30 \mathrm{~kg} / \mathrm{m}^{2}\right)$, with normal weight as reference category. Furthermore, we stratified analyses of morphokinetic parameters for fertilisation method, as a 2-h delay between insemination and fertilisation has been suggested for IVF embryos [36]. In addition, we performed sub-analyses of embryos of couples with only a female subfertility diagnosis (e.g. endometriosis or PCOS) and a male partner with normal semen parameters, or combined female and male factor subfertility. All analyses were performed in SPSS statistics 25.0 (IBM, Armonk, USA) and R (R: A language and Environment for Statistical Computing, version 3.1.3, 2015 for Windows, R Core Team, Vienna, Austria). Two-sided $p$-values $<0.05$ were considered significant. 


\section{Results}

\section{Baseline characteristics}

A total of 268 women ( $n=938$ embryos) were included, of whom 143 were of normal weight ( $n=482$ embryos), 79 overweight ( $n=294$ embryos) and 46 obese $(n=162$ embryos) (Fig. 1). Baseline characteristics were comparable between the three groups, except that normal weight women were more often highly educated than overweight and obese women (62.7, 51.9 and 30.4\%, respectively, $p=0.004$ ) (Table 1 ). Additionally, types of female type subfertility are not significantly different between the three groups (Supplemental table 2).

\section{Morphokinetic parameters}

Linear mixed model analyses showed negative betas for all morphokinetic parameters, indicating a faster development for every increase in BMI point (Table 2). However, this was only significant for tPNf $\left(\beta_{\text {crude }}-0.119 \mathrm{~h}\right.$ (95\%CI -0.206, -0.031), $p=0.008 ; \beta_{\text {adjusted }}-0.091 \mathrm{~h}$ (95\%CI $-0.180,-0.003), p=0.043)$ and $\mathrm{t} 2\left(\beta_{\text {crude }}-0.142 \mathrm{~h}\right.$ (95\%CI $-0.235,-0.049), p=0.003$; $\beta_{\text {adjusted }}-0.111$ h $(95 \% \mathrm{CI}$ -0.205, -0.016), $p=0.022$ ). Stratification for fertilization method demonstrated that the negative beta for $\mathrm{tPNa}$ is almost exclusively based on ICSI embryos (Table 3).
Interestingly, sub-analyses of embryos from couples with female or combined factor subfertility showed an even larger impact of BMI on tPNf $\left(\beta_{\text {crude }}-0.164 \mathrm{~h}\right.$ (95\%CI -0.286, -0.042), $p=0.009 ; \beta_{\text {adjusted }}-0.170 \mathrm{~h}(95 \% \mathrm{CI}$ $-0.293,-0.047), p=0.007)$ and $\mathrm{t} 2\left(\beta_{\text {crude }}-0.194 \mathrm{~h}(95 \% \mathrm{CI}\right.$ $-0.323,-0.064), p=0.004 ; \beta_{\text {adjusted }}-0.199$ h (95\%CI -0.330, -0.067), $p=0.003$ ) (Table 4).

Post-hoc analyses demonstrated a significantly positive beta for $t 8$ in embryos of overweight women, when compared to embryos of normal weight women, $\left(\beta_{\text {crude }}\right.$ $1.744 \mathrm{~h}$ (95\%CI 0.087, 3.401), $p=0.039 ; \beta_{\text {adjusted }} 2.541 \mathrm{~h}$ (95\%CI 0.774, 4.308), $p=0.005$ ) (Table 5). Embryos of obese women reached tPNf and t2 faster than embryos of normal weight women (tPNf: $\beta_{\text {crude }}-1.065 \mathrm{~h}(95 \% \mathrm{CI}$ -2.082, -0.047), $p=0.040$; t2: $\beta_{\text {crude }}-1.311$ h $(95 \% \mathrm{CI}$ $-2.934,-0.227), p=0.018$; t2: $\beta_{\text {adjusted }}-1.101 \mathrm{~h}(95 \% \mathrm{CI}$ $-2.195,-0.008), p=0.048)$.

\section{Implantation potential, predicted by the KIDScore}

The association between maternal BMI and predicted implantation potential, assessed by the KIDScore, was studied by a proportional odds model. The crude model showed a non-significant effect estimate of -0.019 (se $0.015, p=0.206$ ), indicating a lower

Table 1 Baseline characteristics of the VIRTUAL EmbryoScope study population $(n=268)$

\begin{tabular}{|c|c|c|c|c|c|c|c|c|}
\hline & \multirow{2}{*}{\multicolumn{2}{|c|}{$\frac{\text { Normal weight women }}{N=143}$}} & \multirow{2}{*}{\multicolumn{2}{|c|}{$\begin{array}{l}\text { Overweight women } \\
N=79\end{array}$}} & \multicolumn{2}{|c|}{ Obese women } & \multirow[t]{3}{*}{$P$-value } & \multirow[t]{3}{*}{ Missing } \\
\hline & & & & & $N=43$ & & & \\
\hline & Median/N & IQR/\% & Median/N & IQR/\% & Median/N & IQR/\% & & \\
\hline \multicolumn{9}{|l|}{ Maternal factors } \\
\hline Age, years & 34.3 & $30.5-38.3$ & 32.9 & $29.2-36.6$ & 36.3 & $30.3-39.6$ & 0.110 & 0 \\
\hline Geographic origin, Western & 119 & 83.3 & 68 & 86.1 & 37 & 80.4 & 0.709 & 1 \\
\hline Eductional level & & & & & & & 0.004 & 1 \\
\hline Low & 6 & 4.2 & 6 & 7.6 & 5 & 10.9 & & \\
\hline Intermediate & 47 & 33.1 & 27 & 58.7 & 32 & 40.5 & & \\
\hline High & 89 & 62.7 & 41 & 51.9 & 14 & 30.4 & & \\
\hline Folic acid supplements, yes & 135 & 95.1 & 75 & 94.9 & 42 & 91.3 & 0.632 & 1 \\
\hline Vitamins, yes & 80 & 60.6 & 37 & 50.7 & 17 & 41.5 & 0.073 & 22 \\
\hline Alcohol, yes & 63 & 44.4 & 27 & 34.2 & 18 & 39.1 & 0.328 & 1 \\
\hline Cigarettes, yes & 19 & 13.4 & 12 & 15.2 & 7 & 15.2 & 0.914 & 1 \\
\hline \multicolumn{9}{|l|}{ Treatment factors } \\
\hline Cause of subfertility & & & & & & & 0.987 & 0 \\
\hline Female factor & 44 & 30.8 & 22 & 27.8 & 12 & 26.1 & & \\
\hline Male factor & 51 & 35.7 & 30 & 38.0 & 17 & 37.0 & & \\
\hline Combined & 28 & 19.6 & 17 & 21.5 & 9 & 19.6 & & \\
\hline Unexplained & 20 & 14.0 & 10 & 12.7 & 8 & 17.4 & & \\
\hline ICSI, yes & 79 & 55.2 & 50 & 63.3 & 28 & 60.9 & 0.478 & 0 \\
\hline Oocytes aspirated & 8 & $5-12$ & 9 & $6-14$ & 8 & $5-12$ & 0.278 & 2 \\
\hline Ovarian stimulation, GnRH-agonist & 26 & 18.2 & 18 & 22.8 & 12 & 26.1 & 0.459 & 0 \\
\hline
\end{tabular}

Significant differences are depicted in bold

$I Q R$ Interquartile range, ICSI Intracytoplasmic sperm injection 
Table 2 The impact of maternal BMI on morphokinetic parameters

\begin{tabular}{|c|c|c|c|c|c|}
\hline \multirow[t]{2}{*}{ Morphokinetic parameter } & \multicolumn{2}{|l|}{ Crude } & \multicolumn{2}{|l|}{ Adjusted } & \multirow[t]{2}{*}{ Missing } \\
\hline & Beta $(95 \% \mathrm{Cl})$ hours & $p$-value & Beta $(95 \% \mathrm{Cl})$ hours & $p$-value & \\
\hline tPNa & $-0.074(-0.163,0.015)$ & 0.102 & $-0.070(-0.139,-0.001)$ & 0.048 & $448^{\mathrm{a}}$ \\
\hline tPNf & $-0.119(-0.206,-0.031)$ & 0.008 & $-0.091(-0.180,-0.003)$ & 0.043 & 23 \\
\hline t2 & $-0.142(-0.235,-0.049)$ & 0.003 & $-0.111(-0.205,-0.016)$ & 0.022 & 3 \\
\hline t3 & $-0.100(-0.223,0.023)$ & 0.109 & $-0.039(-0.168,0.089)$ & 0.548 & 5 \\
\hline t4 & $-0.122(-0.246,0.001)$ & 0.053 & $-0.087(-0.220,0.047)$ & 0.201 & 8 \\
\hline t5 & $-0.102(-0.266,0.061)$ & 0.220 & $-0.053(-0.229,0.122)$ & 0.549 & 13 \\
\hline t6 & $-0.100(-0.263,0.063)$ & 0.229 & $-0.073(-0.251,0.105)$ & 0.418 & 33 \\
\hline t7 & $-0.069(-0.242 .0 .103)$ & 0.429 & $-0.014(-0.204,0.175)$ & 0.881 & 65 \\
\hline t8 & $-0.002(-0.178,0.174)$ & 0.982 & $0.067(-0.125,0.259)$ & 0.492 & 151 \\
\hline
\end{tabular}

Adjusted for maternal age, fertilization method, type of ovarian stimulation and paternal BMI and age

Significant differences are depicted in bold

$\mathrm{Cl}$ Confidence interval

a In cases of regular IVF, embryos are only transferred to the EmbryoScope after PN inspection, thus tPNa cannot be observed

Table 3 The impact of maternal BMI on morphokinetic parameters, stratified for fertilisation method

\begin{tabular}{|c|c|c|c|c|c|c|c|c|}
\hline \multirow{3}{*}{$\begin{array}{l}\text { Morphokinetic } \\
\text { parameters }\end{array}$} & \multicolumn{4}{|l|}{ IVF $n=111$ women } & \multicolumn{4}{|l|}{ ICSI $n=157$ women } \\
\hline & \multicolumn{2}{|l|}{ Crude } & \multicolumn{2}{|l|}{ Adjusted } & \multicolumn{2}{|l|}{ Crude } & \multicolumn{2}{|l|}{ Adjusted } \\
\hline & Beta $(95 \% \mathrm{Cl})$ hours & $p$-value & Beta $(95 \% \mathrm{Cl})$ hours & $p$-value & Beta $(95 \% \mathrm{Cl})$ hours & $p$-value & Beta $(95 \% \mathrm{Cl})$ hours & $p$-value \\
\hline tPNa ${ }^{a}$ & $0.429(-1.647,2.508)$ & 0.232 & $\mathrm{n} / \mathrm{a}$ & & $-0.070(-0.131,-0.010)$ & 0.024 & $-0.071(-0.140,-0.001)$ & 0.046 \\
\hline tPNf & $-0.103(-0.247,0.041)$ & 0.158 & $-0.115(-0.270,0.040)$ & 0.143 & $-0.091(-0.187,0.005)$ & 0.064 & $-0.080(-0.186,0.026)$ & 0.136 \\
\hline t2 & $-0.152(-0.306,0.002)$ & 0.053 & $-0.146(-0.311,0.019)$ & 0.083 & $-0.095(-0.196,0.007)$ & 0.067 & $-0.146(-0.311,0.019)$ & 0.083 \\
\hline t3 & $-0.085(-0.290,0.119)$ & 0.409 & $-0.046(-0.273,0.180)$ & 0.685 & $-0.071(-0.214,0.073)$ & 0.333 & $-0.031(-0.183,0.121)$ & 0.690 \\
\hline t4 & $-0.101(-0.312,0.109)$ & 0.342 & $-0.086(-0.315,0.143)$ & 0.459 & $-0.105(-0.251,0.042)$ & 0.161 & $0.081(-0.242,0.080)$ & 0.320 \\
\hline t5 & $-0.081(-0.354,0.192)$ & 0.557 & $-0.036(-0.337,0.265)$ & 0.812 & $-0.075(-0.273,0.123)$ & 0.456 & $-0.052(-0.265,0.161)$ & 0.630 \\
\hline t6 & $-0.024(-0.293,0.245)$ & 0.858 & $-0.018(-0.319,0.283)$ & 0.906 & $-0.120(-0.323,0.085)$ & 0.251 & $-0.103(-0.326,0.120)$ & 0.362 \\
\hline t7 & $-0.080(-0.363,0.203)$ & 0.577 & $-0.027(-0.348,0.295)$ & 0.870 & $-0.022(-0.239,0.193)$ & 0.836 & $-0.002(-0.239,0.235)$ & 0.984 \\
\hline t8 & $-0.078(-0.373,0.217)$ & 0.601 & $-0.038(-0.371,0.294)$ & 0.820 & $0.084(-0.132,0.301)$ & 0.443 & $0.122(-0.116,0.360)$ & 0.310 \\
\hline
\end{tabular}

Adjusted for maternal age, type of ovarian stimulation and paternal BMI and age

Significant differences are depicted in bold

$\mathrm{Cl}$ Confidence interval

${ }^{\mathrm{a}} n=5$ for tPNa in IVF-population

KIDScore for a higher BMI. The adjusted model demonstrated a comparable estimate $(\beta-0.020$ (se 0.017), $p=0.218$ ).

Interestingly, sub-analyses of embryos from couples with either a female or a combined factor subfertility diagnosis demonstrated a significant impact of BMI on the KIDScore $\left(\beta_{\text {crude }}-0.049\right.$ (se 0.025), $p=0.052$; $\beta_{\text {adjusted }}-0.073$ (se 0.028 ), $p=0.010$ ). These observations indicate that a higher maternal BMI has a detrimental impact on the predicted implantation potential of embryos of women with an underlying cause for their subfertility.

Post-hoc analyses of maternal BMI into categories also demonstrated non-significant associations between either overweight or obesity and predicted implantation potential ( $\beta_{\text {overweight }} 0.008$ (se $0.014, p=0.547$ ); $\beta_{\text {obesity }}$ -0.253 (se $0.174, p=0.178)$ ). Similar results were found in the adjusted model $\left(\beta_{\text {overweight }} 0.126\right.$ (se 0.192, $p=0.403$ ); $\beta_{\text {obesity }}-0.260$ (se 0.192, $\left.p=0.177\right)$ ).

\section{Pre- and post-transfer clinical treatment outcomes}

Crude linear regression analysis was applied to investigate associations between maternal BMI and the tertiary outcomes. The association between maternal BMI and total number of fertilized oocytes showed that for every point increase in BMI, the total number of fertilized oocytes per patient increased 0.024 (95\%CI -0.075, 0.124, 
Table 4 The impact of maternal BMI on morphokinetic parameters of embryos of couples with either a female factor or combined factor subfertility diagnosis $(n=476)$

\begin{tabular}{|c|c|c|c|c|c|}
\hline \multirow[t]{2}{*}{ Morphokinetic parameter } & \multicolumn{2}{|l|}{ Crude } & \multicolumn{2}{|l|}{ Adjusted } & \multirow[t]{2}{*}{ Missing } \\
\hline & Beta $(95 \% \mathrm{Cl})$ hours & $p$-value & Beta $(95 \% \mathrm{Cl})$ hours & $p$-value & \\
\hline tPNa & $-0.300(-0.256,0.196)$ & 0.790 & $-0.011(-0.120,0.098)$ & 0.841 & 290 \\
\hline tPNf & $-0.164(-0.286,-0.042)$ & 0.009 & $-0.170(-0.293,-0.047)$ & 0.007 & 0 \\
\hline t2 & $-0.194(-0.323,-0.064)$ & 0.004 & $-0.199(-0.330,-0.067)$ & 0.003 & 0 \\
\hline t3 & $-0.169(-0.353,0.015)$ & 0.072 & $-0.097(-0.287,0.092)$ & 0.311 & 0 \\
\hline t4 & $-0.214(-0.397,-0.031)$ & 0.022 & $-0.203(-0.399,-0.008)$ & 0.042 & 0 \\
\hline t5 & $-0.127(-0.358,0.105)$ & 0.280 & $-0.060(-0.308,0.187)$ & 0.629 & 3 \\
\hline t6 & $-0.138(-0.371,0.095)$ & 0.243 & $-0.148(-0.401,0.104)$ & 0.247 & 10 \\
\hline t7 & $-0.148(-0.394,0.099)$ & 0.237 & $-0.097(-0.366,0.173)$ & 0.478 & 27 \\
\hline t8 & $-0.096(-0.358,0.167)$ & 0.472 & $-0.021(-0.316,0.273)$ & 0.887 & 68 \\
\hline
\end{tabular}

Adjusted for maternal age, fertilization method, type of ovarian stimulation and paternal BMI and age

Significant differences are depicted in bold

ClConfidence interval

Table 5 Differences in morphokinetic parameters of embryos from overweight and obese women, compared to embryos of normal weight women

\begin{tabular}{|c|c|c|c|c|c|c|c|c|c|}
\hline \multirow{3}{*}{$\begin{array}{l}\text { Morphokinetic } \\
\text { parameter }\end{array}$} & \multicolumn{4}{|l|}{ Crude } & \multicolumn{4}{|l|}{ Adjusted } & \multirow[t]{3}{*}{ Missing } \\
\hline & \multicolumn{2}{|l|}{ Overweight } & \multicolumn{2}{|l|}{ Obese } & \multicolumn{2}{|l|}{ Overweight } & \multicolumn{2}{|l|}{ Obese } & \\
\hline & $\begin{array}{l}\text { Beta } \\
(95 \% \mathrm{Cl}) \text { hours }\end{array}$ & $p$-value & $\begin{array}{l}\text { Beta } \\
(95 \% \mathrm{Cl}) \text { hours }\end{array}$ & $p$-value & $\begin{array}{l}\text { Beta } \\
(95 \% \mathrm{Cl}) \text { hours }\end{array}$ & $p$-value & $\begin{array}{l}\text { Beta } \\
(95 \% \mathrm{Cl}) \text { hours }\end{array}$ & $p$-value & \\
\hline $\mathrm{tPNa}$ & $\begin{array}{l}-0.593 \\
(-1.438,0.252)\end{array}$ & 0.167 & $\begin{array}{l}-0.404 \\
(-1.422,0.614)\end{array}$ & 0.433 & $\begin{array}{l}-0.316 \\
(-0.981,0.349)\end{array}$ & 0.348 & $\begin{array}{l}-0.628 \\
(-1.401,0.144)\end{array}$ & 0.110 & 448 \\
\hline tPNf & $\begin{array}{l}-0.737 \\
(-1.588,0.114)\end{array}$ & 0.089 & $\begin{array}{l}-1.065 \\
(-2.082,-0.047)\end{array}$ & 0.040 & $\begin{array}{l}-0.127 \\
(-0.984,0.730)\end{array}$ & 0.770 & $\begin{array}{l}-0.914 \\
(-1.940,0.111)\end{array}$ & 0.080 & 23 \\
\hline t2 & $\begin{array}{l}-0.630 \\
(-1.533,0.273)\end{array}$ & 0.171 & $\begin{array}{l}-1.311 \\
(-2.394,-0.227)\end{array}$ & 0.018 & $\begin{array}{l}-0.050 \\
(-0.955,0.856)\end{array}$ & 0.914 & $\begin{array}{l}-1.101 \\
(-2.195,-0.008)\end{array}$ & 0.048 & 3 \\
\hline t3 & $\begin{array}{l}-0.395 \\
(-1.583,0.794)\end{array}$ & 0.514 & $\begin{array}{l}-0.852 \\
(-2.278,0.573)\end{array}$ & 0.240 & $\begin{array}{l}0.352 \\
(-0.876,1.580)\end{array}$ & 0.573 & $\begin{array}{l}-0.228 \\
(-1.711,1.254)\end{array}$ & 0.762 & 5 \\
\hline t4 & $\begin{array}{l}-0.768 \\
(-1.969,0.434)\end{array}$ & 0.210 & $\begin{array}{l}-0.825 \\
(-2.267,0.617)\end{array}$ & 0.261 & $\begin{array}{l}-0.239 \\
(-1.523,1.044)\end{array}$ & 0.713 & $\begin{array}{l}-0.418 \\
(-1.966,1.131)\end{array}$ & 0.596 & 8 \\
\hline t5 & $\begin{array}{l}0.571 \\
(-0.989,2.131)\end{array}$ & 0.472 & $\begin{array}{l}-0.626 \\
(-3.501,0.249)\end{array}$ & 0.089 & $\begin{array}{l}1.331 \\
(-0.319,2.982)\end{array}$ & 0.113 & $\begin{array}{l}-1.080 \\
(-3.079,0.918)\end{array}$ & 0.288 & 13 \\
\hline t6 & $\begin{array}{l}0.510 \\
(-1.039,2.059)\end{array}$ & 0.517 & $\begin{array}{l}-1.644 \\
(-3.526,0.237)\end{array}$ & 0.086 & $\begin{array}{l}1.191 \\
(-0.470,2.852)\end{array}$ & 0.159 & $\begin{array}{l}-1.446 \\
(-3.479,0.587)\end{array}$ & 0.162 & 33 \\
\hline t7 & $\begin{array}{l}0.942 \\
(-0.683,2.566)\end{array}$ & 0.542 & $\begin{array}{l}-0.378 \\
(-3.353,0.598)\end{array}$ & 0.171 & $\begin{array}{l}1.672 \\
(-2.996,1.264)\end{array}$ & 0.060 & $\begin{array}{l}-0.866 \\
(-2.996,1.264)\end{array}$ & 0.424 & 65 \\
\hline t8 & $\begin{array}{l}1.744 \\
(0.087,3.401)\end{array}$ & 0.039 & $\begin{array}{l}-0.994 \\
(-2.977,0.989)\end{array}$ & 0.324 & $\begin{array}{l}2.541 \\
(0.774,4.308)\end{array}$ & 0.005 & $\begin{array}{l}-0.235 \\
(-2.367,1.896)\end{array}$ & 0.828 & 151 \\
\hline
\end{tabular}

Adjusted for maternal age, fertilization method, type of ovarian stimulation and paternal BMI and age Significant differences are depicted in bold

Cl Confidence interval

$p=0.630$ ), yet this was not significant (Table 6). Maternal BMI was also not significantly associated with other pre- or post-transfer clinical treatment outcomes, such as implantation rate (odds ratio 0.994 (95\%CI 0.936, 1.054), $p=0.994)$.
Post-hoc analyses of pre- and post-transfer clinical treatment outcomes of overweight and obese women as separate groups demonstrated no significant associations, when compared to normal weight women (Tables 7 and 8). 
Table 6 The impact of maternal BMI on IVF/ICSI treatment outcome parameters

\begin{tabular}{|c|c|c|c|c|}
\hline & Crude & & Adjusted & \\
\hline Pre-transfer & Beta $(95 \% \mathrm{Cl})$ & $p$-value & Beta $(95 \% \mathrm{Cl})$ & $p$-value \\
\hline Total fertilized oocytes & $0.024(-0.075,0.124)$ & 0.630 & $0.031(-0.067,0.129)$ & 0.532 \\
\hline Fertilization rate & $-0.003(-0.009,0.003)$ & 0.329 & $-0.003(-0.009,0.003)$ & 0.303 \\
\hline Total usable embryos & $0.018(-0.049,0.085)$ & 0.605 & $0.021(-0.046,0.088)$ & 0.536 \\
\hline Usage rate & $0.000(-0.007,0.007)$ & 0.913 & $0.000(-0.007,0.007)$ & 0.928 \\
\hline Post-transfer & OR $(95 \% \mathrm{Cl})$ & $p$-value & OR $(95 \% \mathrm{Cl})$ & $p$-value \\
\hline Positive $\beta$-hCG-test $n=106$ & $0.994(0.936,1.054)$ & 0.994 & $0.997(0.938,1.060)$ & 0.930 \\
\hline Gestational sac $n=97$ & $0.997(0.939,1.059)$ & 0.923 & $1.000(0.940,1.064)$ & 0.998 \\
\hline Fetal heartbeat $n=90$ & $0.985(0.927,1.047)$ & 0.630 & $0.986(0.926,1.050)$ & 0.663 \\
\hline Live born ${ }^{\mathrm{a}} n=61$ & $0.998(0.92,1.069)$ & 0.949 & $1.000(0.932,1.073)$ & 0.992 \\
\hline Cumulative pregnancy $^{b} n=132$ & $1.036(0.977,1.097)$ & 0.238 & $1.044(0.983,1.109)$ & 0.163 \\
\hline
\end{tabular}

Adjusted for maternal age and type of ovarian stimulation $\mathrm{Cl}$ Confidence interval, OR Odds ratio

${ }^{\mathrm{a}}$ missing $n=22^{\mathrm{b}}$ missing $n=8$

Table 7 Post-transfer treatment outcome parameters per BMI category

\begin{tabular}{|c|c|c|c|c|c|c|c|}
\hline & \multicolumn{2}{|c|}{$\begin{array}{l}\text { Normal weight women } \\
n=143\end{array}$} & \multicolumn{2}{|c|}{$\begin{array}{l}\text { Overweight women } \\
n=79\end{array}$} & \multicolumn{2}{|c|}{$\begin{array}{l}\text { Obese women } \\
n=46\end{array}$} & \multirow[t]{2}{*}{$P$-value } \\
\hline & $\mathbf{N}$ & $\%$ & $\mathbf{N}$ & $\%$ & $\mathbf{N}$ & $\%$ & \\
\hline Positive $\beta$-hCG-test & 70 & $43.8 \%$ & 43 & $49.4 \%$ & 20 & $35.1 \%$ & 0.237 \\
\hline Gestational sac & 62 & $38.8 \%$ & 41 & $47.1 \%$ & 18 & $31.6 \%$ & 0.163 \\
\hline Fetal heartbeat & 58 & $36.3 \%$ & 36 & $41.4 \%$ & 17 & $29.8 \%$ & 0.369 \\
\hline Live born ${ }^{\mathrm{a}}$ & 36 & $25.2 \%$ & 28 & $34.1 \%$ & 12 & $23.1 \%$ & 0.257 \\
\hline
\end{tabular}

${ }^{a}$ missing $n=22$

Table 8 Differences in treatment outcome parameters for overweight and obese women, compared to normal weight women

\begin{tabular}{|c|c|c|c|c|c|c|c|c|}
\hline \multirow[b]{3}{*}{ Pre-transfer } & \multicolumn{4}{|l|}{ Crude } & \multicolumn{4}{|l|}{ Adjusted } \\
\hline & \multicolumn{2}{|l|}{ Overweight } & \multicolumn{2}{|l|}{ Obese } & \multicolumn{2}{|l|}{ Overweight } & \multicolumn{2}{|l|}{ Obese } \\
\hline & Beta $(95 \% \mathrm{Cl})$ & $p$-value & Beta $(95 \% \mathrm{Cl})$ & $p$-value & Beta $(95 \% \mathrm{Cl})$ & $p$-value & Beta $(95 \% \mathrm{Cl})$ & $p$-value \\
\hline $\begin{array}{l}\text { Total fertilized } \\
\text { oocytes }\end{array}$ & $0.541(-0.420,1.501)$ & 0.269 & $0.183(-0.969,1.335)$ & 0.755 & $0.428(-0.524,1.379)$ & 0.377 & $0.315(-0.824,1.455)$ & 0.586 \\
\hline Fertilization rate & $-0.019(-0.079,0.041)$ & 0.542 & $-0.025(-0.097,0.047)$ & 0.491 & $-0.015(-0.076,0.045)$ & 0.617 & $-0.029(-0.101,0.044)$ & 0.434 \\
\hline Total usable embryos & $0.351(-0.298,1.000)$ & 0.288 & $0.097(-0.681,0.876)$ & 0.805 & $0.292(-0.357,0.940)$ & 0.377 & $0.164(-0.612,0.941)$ & 0.677 \\
\hline Usage rate & $-0.029(-0.097,0.038)$ & 0.394 & $0.012(-0.070,0.093)$ & 0.777 & $-0.026(-0.095,0.042)$ & 0.446 & $0.010(-0.072,0.092)$ & 0.815 \\
\hline Post-transfer & OR $(95 \% \mathrm{Cl})$ & $p$-value & OR $(95 \% \mathrm{Cl})$ & $p$-value & OR $(95 \% \mathrm{Cl})$ & $p$-value & OR $(95 \% \mathrm{Cl})$ & $p$-value \\
\hline Positive $\beta$-hCG-test & $1.349(0.750,2.428)$ & 0.318 & $0.843(0.420,1.693)$ & 0.632 & $1.296(0.705,2.380)$ & 0.404 & $0.895(0.435,1.842)$ & 0.763 \\
\hline Gestational sac & $1.459(0.807,2.640)$ & 0.211 & $0.931(0.459,1.888)$ & 0.843 & $1.392(0.754,2.752)$ & 0.291 & $0.986(0.473,2.053)$ & 0.970 \\
\hline Fetal heartbeat & $1.191(0.654,2.170)$ & 0.567 & $0.872(0.426,1.785)$ & 0.709 & $1.114(0.600,2.068)$ & 0.733 & $0.908(0.433,1.902)$ & 0.798 \\
\hline Live born ${ }^{\mathrm{a}}$ & $1.303(0.665,2.552)$ & 0.440 & $1.051(0.469,2.354)$ & 0.904 & $1.254(0.632,2.491)$ & 0.517 & $1.125(0.494,2.560)$ & 0.780 \\
\hline $\begin{array}{l}\text { Cumulative } \\
\text { pregnancy }\end{array}$ & $1.446(0.823,2.540)$ & 0.199 & $1.229(0.663,2.545)$ & 0.446 & $1.304(0.723,2.350)$ & 0.378 & $1.510(0.742,3.074)$ & 0.256 \\
\hline
\end{tabular}

Adjusted for maternal age and type of ovarian stimulation

Cl Confidence interval, OR Odds ratio

${ }^{\mathrm{a}}$ missing $n=22^{\mathrm{b}}$ missing $n=8$ 


\section{Discussion}

\section{Summary of findings}

This study aimed to investigate the hypothesis that an elevated BMI in women undergoing IVF/ICSI treatment has a detrimental impact on 1) preimplantation morphokinetic parameters until day 3 of development, 2) predicted implantation potential and 3) pre- and posttransfer clinical treatment outcomes. We observed that a higher maternal BMI is associated with a faster progression through the cleavage stages. No significant association of maternal BMI with predicted implantation potential, as assessed by the KIDScore algorithm, was shown. However, in embryos of couples with female or combined factor subfertility, maternal BMI was associated with faster early embryonic development and lower predicted implantation potential. In addition, no significant associations were shown between maternal BMI and the tertiary clinical treatment outcomes.

When maternal BMI was divided into categories, we observed delayed reaching of the 8-cell stage in embryos of overweight women, whereas embryos of obese women reach the 2-cell stage faster than embryos of normal weight women. Morphokinetic embryonic quality and clinical treatment outcomes were comparable between the three groups.

\section{Interpretation}

In the first study investigating the impact of maternal BMI on morphokinetic parameters, embryos of normal weight and obese infertile donors developed comparably [29]. However, recently a delay in late cleavage divisions $(t 5, t 8)$ was shown for embryos of overweight and obese women, which is (partially) in contrast to our findings [28]. This study is not directly comparable to ours, as ICSI cycles were studied exclusively, whereas we studied both IVF and ICSI cycles. This may have direct implications, by differences in fertilization techniques, as well as indirect, by differences in study population. In contrast to the study of Bartolacci et al., Leary et al. reported that embryos of overweight and obese women reach the morula stage, and subsequently the blastocyst stage, faster than embryos of normal weight women, although these embryos also have a higher rate of cleavage-stage arrest [35]. Although it is beyond the scope of the current study, the impact of maternal BMI on blastocyst formation rate, an important predictor for implantation, remains inconclusive. Some report no impact or negative impact of high maternal BMI on blastocyst formation, whereas a recent large study reports a higher blastocyst formation rate for obese women [35, 37-41].

It is hypothesized that maternal adiposity may have an effect prior to fertilization. The altered metabolic environment, as a result of an imbalanced diet and chronic excessive oxidative stress, contributes to an abnormal follicular microenvironment [20, 42]. This aberrant microenvironment can derange several pathways including the one-carbon metabolism, which is important for numerous processes involved in reproduction, such as protein and DNA synthesis and redox regulation [43]. This hypothesis is supported by mouse studies showing an effect of obesity on oocyte polarization, reactive oxygen species levels and DNA methylation, including methylation of metabolism-related genes, such as the leptin promotor region $[44,45]$. In humans, it has been demonstrated that rising BMI affects regulation of oocyte RNA expression and oocyte metabolism [35, 46, 47]. Furthermore, the maternally-inherited genome passively demethylates with each cell-division, reaching the lowest level at the blastocyst stage, whereas the paternally-inherited genome actively demethylates within $8 \mathrm{~h}$ after fertilization [48-50]. Similarly, studies of human preimplantation embryos show that oocyte molecular programs are gradually degraded during the first 3 days after fertilization and those of the embryo genome are activated, culminating between the 4- and 8-cell stage [51-53]. This suggests that early preimplantation embryonic development is primarily driven by the maternal (epi-)genome.

A common cause of female subfertility is polycystic ovarian syndrome (PCOS), which is associated with obesity. PCOS is characterized by a combination of polycystic ovaries, hyperadrogenism and anovulation $[54,55]$. Research in women with PCOS undergoing IVF/ICSI shows impaired developmental competence of oocytes, yet preimplantation embryonic development is unaffected [56-59]. In our study, sub-analyses of embryos of women with PCOS demonstrated no significant impact of BMI on morphokinetic parameters (data not shown).

Interestingly, the differences in individual morphokinetic parameters did not translate into differences in the KIDScore distribution. Although the KIDScore is based on only a limited number of parameters, the impact of maternal BMI on these parameters may be too small to induce a shift in the distribution of KIDScores. In embryos of couples with female or combined factor subfertility, however, we found a larger impact of BMI on individual morphokinetic parameters, which may explain the significant negative impact of maternal BMI on the KIDScore. As this is the first study to investigate the impact of maternal BMI on a morphokinetic quality score, comparison to other studies is limited. The KIDScore is a widely applicable morphokinetic (de)selection tool, as it ranks embryo's according to their implantation potential, regardless of the fertilisation technique used and culture conditions applied [25]. Furthermore, it has 
a high blastulation predictability and performs superior to conventional morphology evaluation for predicting live births, when applied to day 3 embryos [60]. However, morphokinetic based embryo selection may not be accessible for all fertility clinics, as time-lapse imaging is a relatively expensive technique when compared to conventional culture. In line with this, there are several studies that have investigated the impact of maternal BMI on conventional morphological quality. Although these studies differ in terms of parameters of morphological quality and statistical methods, only one demonstrated an impact of maternal BMI on embryo morphology, suggesting that the impact of maternal BMI on embryonic quality is relatively small $[18,21,28,61,62]$.

As a tertiary outcome we have addressed the impact of maternal BMI on clinical treatment outcomes in our dataset and found no significant associations. It is very likely that the absence of significant findings can be explained by a lack of power. Yet, the detrimental impact of maternal BMI on success rates of IVF/ICSI treatment has been widely shown in other studies. A recent metaanalysis of over 600,000 women reported a $15 \%$ smaller chance of a live birth after IVF/ICSI treatment for obese women compared to normal weight women [63]. Moreover, an additional factor in post-transfer outcomes is the uterine environment. A large retrospective study of over 9,500 normal weight oocyte donors reported lower success rates for obese recipients than for normal weight recipients [64]. Although the exact mechanisms by which obesity alters endometrial receptivity are poorly understood, it is suggested that decidualisation is impaired by genetic dysregulation [65-67].

\section{Strengths and limitations}

By applying a standardized method to measure BMI prior to IVF/ICSI treatment, instead of relying on selfreported data, we reduced the risk of response bias. Moreover, BMI was also categorized according to the World Health Organization classification to facilitate comparison between studies. Statistical strengths are the application of linear mixed model analyses, which takes the clustering-effect of multiple embryos from one women into account, and adjustments for important treatment factors and paternal factors such as age and BMI, so that maternal effects could be studied independently. Another strength is the use of the KIDScore to evaluate embryonic morphokinetic quality at day 3 after fertilization. This deselection tool is universally applicable and has area under the curve of 0.65 for prediction of implantation, which can be considered as a fair predictor [25].

The main limitation of our study is that we have only data until day 3 of development and not until the blastocyst stage, as this is associated with higher rates of pregnancy and live birth [68]. This study was conducted in a time in which fresh transfer of cleavage embryos was routine care in most IVF clinics, including ours, but future research should include embryonic development until day 5. Also, due to the inclusion of IVF treatments, the moment of pronuclear appearance could not be observed in these cases. Although the diverse study population, increases the generalizability of our results, it can also be considered a weakness. As it included both IVF and ICSI treatments and different stimulation protocols, it is a source of possible bias and may elicit divergent results. Furthermore, it is standard care at our clinic to only perform IVF/ICSI treatment in women with a $\mathrm{BMI}<34 \mathrm{~kg} / \mathrm{m}^{2}$, as IVF/ICSI treatment in women with a higher BMI is rarely feasible and associated with increased pregnancy complications [69]. Nonetheless, this practice induces a selection bias for this study. Also, our study population did not comprise any women with underweight. This limited the possibilities to investigate the impact of the full range of maternal BMI. Finally, this study was performed at a tertiary university based hospital. Although not all subfertile couples were in need of tertiary referral or care, our results cannot be automatically extrapolated to the general subfertile population, which may have consequences for the external validity of this study.

\section{Conclusions}

In this study we show that maternal BMI is positively associated with faster progression through the pronuclear and early cleavage stages and negatively with embryo implantation potential. So far, and very likely due to lack of power no associations between maternal BMI and clinical treatment outcomes were observed.

Overweight and obesity are complex diseases and often the result of the interplay between nutrition, lifestyle and genetics. Future research is needed to elucidate the pathophysiological processes involved in the effects of maternal BMI on preimplantation development. Possible explanations might be found in alterations in oocyte quality, DNA damage and decreased cytoplasmic quality, as we observed an impact of maternal BMI on embryo quality in couples with female or combined factor subfertility. In addition, potential metabolic alterations underlying the observed differences in preimplantation development may also have consequences for post-implantation development. Although not demonstrated in this study, the negative effect of increased BMI on ART treatment outcomes has been widely reported. Moreover, maternal overweight and obesity have serious implications for pregnancy outcome and offspring health. Therefore, it is 
recommended to optimise lifestyle to achieve a healthy weight prior to IVF/ICSI treatment, for example by effective eHealth coaching programs [70, 71]. A healthy weight maximises the general efficiency of the treatment is and minimises alterations in the (early) development of the future generation are minimised.

\section{Abbreviations}

AMH: Anti-Müllerian hormone; BMI: Body mass index; Cl: Confidence interval; ET: Embryo transfer; FSH: Follicle stimulating hormone; GnRH: Gonadotrophinreleasing hormone; hCG: Human chorionic gonadotrophin; ICC: Intraclass correlation coefficient; ICSI: Intracytoplasmic sperm injection; IVF: In vitro fertilization; OR: Odds ratio; PCOS: Polycystic ovarian syndrome; Pna: Pronuclear appearance; PNf: Pronuclear fading.

\section{Supplementary Information}

The online version contains supplementary material available at https://doi. org/10.1186/s12958-021-00822-0.

Additional file 1: Supplemental table 1. Morpokinetic parameters of the KIDScore algorithm. Supplemental table 2. Female type of subfertility, stratified for maternal BMI.

\section{Acknowledgements}

We gratefully acknowledge the Rotterdam Periconception Cohort team for data acquisition. Furthermore, we thank all staff members from the outpatient Fertility clinic and laboratory and the participating couples, gynecologists, and midwifery practices in Rotterdam for their contributions.

\section{Authors' contributions}

EB and RST initiated the research question and supervised all aspects of the study. JL was responsible for the IVF and ICSI patients. EB was responsible for time-lapse imaging. LvD, JH, EB and EvM contributed to data acquisition. SW and MR initiated and supervised the statistical procedures of the manuscript. $L v D, M R$, and EB wrote the first draft. All authors contributed to the writing and the critical revisions of the manuscript and all authors approved the final version of the manuscript and authorized the submitted version.

\section{Funding}

This research was funded by the Department of Obstetrics and Gynecology of the Erasmus MC, University Medical Center, Rotterdam, the Netherlands, and the Erasmus MC Medical Research Advisor Committee's 'Health Care Efficiency Research' program (OZBS72.16080).

\section{Availability of data and materials}

The datasets used and/or analysed during the current study are available from the corresponding author on reasonable request.

\section{Declarations}

\section{Ethics approval and consent to participate}

This study was conducted according to the guidelines laid down in the Declaration of Helsinki and approved by the Medical Ethical and Institutional Review Board of the Erasmus MC, University Medical Center, Rotterdam, The Netherlands (MEC-2004-227).

\section{Consent for publication}

Written informed consent was obtained from all women at enrollment.

\section{Competing interests}

Dr. Laven reports grants and personal fees from Ferring, personal fees from Titus Healthcare, grants and personal fees from Ansh Labs, grants from $\mathrm{NIH}$, grants from Dutch Heart Association, grants from ZonMW, outside the submitted work. None of the other authors have a conflict of interest.

\section{Author details}

${ }^{1}$ Department of Obstetrics and Gynecology, Erasmus MC, University Medical Center, Dr. Molewaterplein 40, Rotterdam 3015 GD, The Netherlands. ' ${ }^{2}$ Department of Biostatistics, Erasmus MC, University Medical Center, Rotterdam, The Netherlands. ${ }^{3}$ Division of Reproductive Endocrinology and Infertility, Department of Obstetrics and Gynecology, Erasmus MC, University Medical Center, Rotterdam, The Netherlands. ${ }^{4}$ Department of Developmental Biology, Erasmus MC, University Medical Center, Rotterdam, The Netherlands.

Received: 4 April 2021 Accepted: 30 August 2021

Published online: 18 September 2021

\section{References}

1. Ng M, Fleming T, Robinson M, Thomson B, Graetz N, Margono C, et al. Global, regional, and national prevalence of overweight and obesity in children and adults during 1980-2013: a systematic analysis for the Global Burden of Disease Study 2013. Lancet. 2014;384(9945):766-81.

2. Marti A, Martinez-Gonzalez MA, Martinez JA. Interaction between genes and lifestyle factors on obesity. Proc Nutr Soc. 2008;67(1):1-8.

3. Wright SM, Aronne LJ. Causes of obesity. Abdom Imaging. 2012;37(5):730-2.

4. Redinger RN. The pathophysiology of obesity and its clinical manifestations. Gastroenterol Hepatol (N Y). 2007;3(11):856-63.

5. Calle EE, Kaaks R. Overweight, obesity and cancer: epidemiological evidence and proposed mechanisms. Nat Rev Cancer. 2004;4(8):579-91.

6. Mokdad AH, Ford ES, Bowman BA, Dietz WH, Vinicor F, Bales VS, et al. Prevalence of obesity, diabetes, and obesity-related health risk factors, 2001. JAMA. 2003;289(1):76-9.

7. Overweight and obesity - BMl statistics: Eurostat; 2020 [updated 4/23/2020]. Available from: https://ec.europa.eu/eurostat/statistics-explained/index. php/Overweight and obesity - BMI_statistics. Accessed 21 Dec 2020

8. Lashen H, Fear K, Sturdee DW. Obesity is associated with increased risk of first trimester and recurrent miscarriage: matched case-control study. Hum Reprod. 2004;19(7):1644-6.

9. van der Steeg JW, Steures P, Eijkemans MJ, Habbema JD, Hompes PG, Burggraaff JM, et al. Obesity affects spontaneous pregnancy chances in subfertile, ovulatory women. Hum Reprod. 2008;23(2):324-8.

10. Dag ZO, Dilbaz B. Impact of obesity on infertility in women. J Turk Ger Gynecol Assoc. 2015;16(2):111-7.

11. Watson RR. Handbook of fertility: nutrition, diet, lifestyle and reproductive health. In: Watson RR, editor. 1st ed. Cambridge: Academic; 2015; 2015. p. 120-7.

12. Spandorfer SD, Kump L, Goldschlag D, Brodkin T, Davis OK, Rosenwaks Z. Obesity and in vitro fertilization: negative influences on outcome. J Reprod Med. 2004;49(12):973-7.

13. Fedorcsak P, Storeng R, Dale PO, Tanbo T, Abyholm T. Obesity is a risk factor for early pregnancy loss after IVF or ICSI. Acta Obstet Gynecol Scand. 2000;79(1):43-8.

14. Moragianni VA, Jones SM, Ryley DA. The effect of body mass index on the outcomes of first assisted reproductive technology cycles. Fertil Steril. 2012;98(1):102-8

15. Metwally M, Li TC, Ledger WL. The impact of obesity on female reproductive function. Obes Rev. 2007:8(6):515-23.

16. Valckx SD, De Pauw I, De Neubourg D, Inion I, Berth M, Fransen E, et al. BMI-related metabolic composition of the follicular fluid of women undergoing assisted reproductive treatment and the consequences for oocyte and embryo quality. Hum Reprod. 2012;27(12):3531-9.

17. Bellver J, Melo MA, Bosch E, Serra V, Remohi J, Pellicer A. Obesity and poor reproductive outcome: the potential role of the endometrium. Fertil Steril. 2007;88(2):446-51.

18. Metwally M, Cutting R, Tipton A, Skull J, Ledger WL, Li TC. Effect of increased body mass index on oocyte and embryo quality in IVF patients. Reprod Biomed Online. 2007;15(5):532-8.

19. Song J, Xiang S, Pang C, Guo J, Sun Z. Metabolomic alternations of follicular fluid of obese women undergoing in-vitro fertilization treatment. Sci Rep. 2020;10(1):5968.

20. Robker RL, Akison LK, Bennett BD, Thrupp PN, Chura LR, Russell DL, et al. Obese women exhibit differences in ovarian metabolites, hormones, 
and gene expression compared with moderate-weight women. J Clin Endocrinol Metab. 2009;94(5):1533-40

21. Sathya A, Balasubramanyam S, Gupta S, Verma T. Effect of body mass index on in vitro fertilization outcomes in women. J Hum Reprod Sci. 2010;3(3):135-8.

22. Zander-Fox DL, Henshaw R, Hamilton H, Lane M. Does obesity really matter? The impact of BMl on embryo quality and pregnancy outcomes after IVF in women aged $\leq 38$ years. Aust N Z J Obstet Gynaecol. 2012;52(3):270-6.

23. Carrell DT, Jones KP, Peterson CM, Aoki V, Emery BR, Campbell BR. Body mass index is inversely related to intrafollicular HCG concentrations, embryo quality and IVF outcome. Reprod Biomed Online. 2001;3(2):109-11.

24. Payne D, Flaherty SP, Barry MF, Matthews CD. Preliminary observations on polar body extrusion and pronuclear formation in human oocytes using time-lapse video cinematography. Hum Reprod. 1997;12(3):532-41.

25. Petersen BM, Boel M, Montag M, Gardner DK. Development of a generally applicable morphokinetic algorithm capable of predicting the implantation potential of embryos transferred on Day 3. Hum Reprod. 2016:31(10):2231-44.

26. Freour T, Dessolle L, Lammers J, Lattes S, Barriere P. Comparison of embryo morphokinetics after in vitro fertilization-intracytoplasmic sperm injection in smoking and nonsmoking women. Fertil Steril. 2013;99(7):1944-50

27. Wissing ML, Bjerge MR, Olesen Al, Hoest T, Mikkelsen AL. Impact of PCOS on early embryo cleavage kinetics. Reprod Biomed Online. 2014;28(4):508-14.

28. Bartolacci A, Buratini J, Moutier C, Guglielmo MC, Novara PV, Brambillasca $F$, et al. Maternal body mass index affects embryo morphokinetics: a timelapse study. J Assist Reprod Genet. 2019;36(6):1109-16.

29. Bellver J, Mifsud A, Grau N, Privitera L, Meseguer M. Similar morphokinetic patterns in embryos derived from obese and normoweight infertile women: a time-lapse study. Hum Reprod. 2013;28(3):794-800.

30. Steegers-Theunissen RP, Verheijden-Paulissen JJ, van Uitert EM, Wildhagen MF, Exalto N, Koning AH, et al. Cohort profile: the Rotterdam periconceptional cohort (predict study). Int J Epidemiol. 2016;45(2):374-81.

31. Hohmann FP, Macklon NS, Fauser BC. A randomized comparison of two ovarian stimulation protocols with gonadotropin-releasing hormone $(\mathrm{GnRH})$ antagonist cotreatment for in vitro fertilization commencing recombinant follicle-stimulating hormone on cycle day 2 or 5 with the standard long GnRH agonist protocol. J Clin Endocrinol Metab. 2003:88(1):166-73.

32. Heijnen EM, Eijkemans MJ, De Klerk C, Polinder S, Beckers NG, Klinkert ER, et al. A mild treatment strategy for in-vitro fertilisation: a randomised non-inferiority trial. Lancet. 2007;369(9563):743-9.

33. Dhont M, editor. WHO-classification of anovulation: background, evidence and problems. International Congress Series. Amsterdam: Elsevier; 2005.

34. Ciray HN, Campbell A, Agerholm IE, Aguilar J, Chamayou S, Esbert M, et al. Proposed guidelines on the nomenclature and annotation of dynamic human embryo monitoring by a time-lapse user group. Hum Reprod. 2014;29(12):2650-60.

35. Leary C, Leese HJ, Sturmey RG. Human embryos from overweight and obese women display phenotypic and metabolic abnormalities. Hum Reprod. 2015;30(1):122-32.

36. Bodri D, Sugimoto T, Serna JY, Kondo M, Kato R, Kawachiya S, et al. Influence of different oocyte insemination techniques on early and late morphokinetic parameters: retrospective analysis of 500 time-lapse monitored blastocysts. Fertil Steril. 2015;104(5):1175-81.e1-2.

37. Rittenberg V, Sobaleva S, Ahmad A, Oteng-Ntim E, Bolton V, Khalaf Y, et al. Influence of BMI on risk of miscarriage after single blastocyst transfer. Hum Reprod. 2011;26(10):2642-50.

38. Sarais V, Pagliardini L, Rebonato G, Papaleo E, Candiani M, Vigano P. A comprehensive analysis of body mass index effect on in vitro fertilization outcomes. Nutrients. 2016;8(3):109.

39. Zhang JJ, Feret M, Chang L, Yang M, Merhi Z. Obesity adversely impacts the number and maturity of oocytes in conventional IVF not in minimal stimulation IVF. Gynecol Endocrinol. 2015;31(5):409-13.

40. Kim J, Patounakis G, Juneau C, Morin S, Neal S, Bergh P, et al. The Appraisal of Body Content (ABC) trial: increased male or female adiposity does not significantly impact in vitro fertilization laboratory or clinical outcomes. Fertil Steril. 2021;116(2):444-52.

41. Comstock IA, Kim S, Behr B, Lathi RB. Increased body mass index negatively impacts blastocyst formation rate in normal responders undergoing in vitro fertilization. J Assist Reprod Genet. 2015;32(9):1299-304.

42. Ruebel ML, Piccolo BD, Mercer KE, Pack L, Moutos D, Shankar K, et al. Obesity leads to distinct metabolomic signatures in follicular fluid of women undergoing in vitro fertilization. Am J Physiol Endocrinol Metab. 2019:316(3):E383-96.

43. Steegers-Theunissen RP, Twigt J, Pestinger V, Sinclair KD. The periconceptional period, reproduction and long-term health of offspring: the importance of one-carbon metabolism. Hum Reprod Update. 2013;19(6):640-55.

44. Hou YJ, Zhu CC, Duan X, Liu HL, Wang Q, Sun SC. Both diet and gene mutation induced obesity affect oocyte quality in mice. Sci Rep. 2016;6:18858.

45. Ge ZJ, Luo SM, Lin F, Liang QX, Huang L, Wei YC, et al. DNA methylation in oocytes and liver of female mice and their offspring: effects of high-fatdiet-induced obesity. Environ Health Perspect. 2014;122(2):159-64.

46. Ruebel ML, Cotter M, Sims CR, Moutos DM, Badger TM, Cleves MA, et al. Obesity modulates inflammation and lipid metabolism oocyte gene expression: a single-cell transcriptome perspective. J Clin Endocrinol Metab. 2017;102(6):2029-38.

47. Llonch S, Barragan M, Nieto P, Mallol A, Elosua-Bayes M, Lorden P, et al. Single human oocyte transcriptome analysis reveals distinct maturation stage-dependent pathways impacted by age. Aging Cell. 2021:20(5):e13360.

48. Mayer W, Fandele R, HaafT. Spatial separation of parental genomes during mouse interspecific (Mus musculus $\times$ M. spretus) spermiogenesis. Chromosome Res. 2000;8(6):555-8.

49. Howlett SK, Reik W. Methylation levels of maternal and paternal genomes during preimplantation development. Development. 1991;113(1):119-27.

50. Rivera RM, Ross JW. Epigenetics in fertilization and preimplantation embryo development. Prog Biophys Mol Biol. 2013;113(3):423-32.

51. Niakan KK, Han J, Pedersen RA, Simon C, Pera RA. Human pre-implantation embryo development. Development. 2012;139(5):829-41.

52. Braude P, Bolton V, Moore S. Human gene expression first occurs between the four- and eight-cell stages of preimplantation development. Nature. 1988;332(6163):459-61

53. Tesarik J, Kopecny V, Plachot M, Mandelbaum J. High-resolution autoradiographic localization of DNA-containing sites and RNA synthesis in developing nucleoli of human preimplantation embryos: a new concept of embryonic nucleologenesis. Development. 1987;101(4):777-91.

54. Rotterdam EA-SPCWG. Revised 2003 consensus on diagnostic criteria and long-term health risks related to polycystic ovary syndrome. Fertil Steril. 2004;81(1):19-25.

55. Steegers-Theunissen RPM, Wiegel RE, Jansen PW, Laven JSE, Sinclair KD. Polycystic ovary syndrome: a brain disorder characterized by eating problems originating during puberty and adolescence. Int J Mol Sci. 2020;21(21):8211

56. Dumesic DA, Abbott DH. Implications of polycystic ovary syndrome on oocyte development. Semin Reprod Med. 2008;26(1):53-61.

57. Sundvall L, Kirkegaard K, Ingerslev HJ, Knudsen UB. Unaltered timing of embryo development in women with polycystic ovarian syndrome (PCOS): a time-lapse study. J Assist Reprod Genet. 2015;32(7):1031-42.

58. Tam Le M, Van Nguyen T, Thanh Nguyen T, Thanh Thi Nguyen T, An Thi Nguyen T, Huy Vu Nguyen Q, et al. Does polycystic ovary syndrome affect morphokinetics or abnormalities in early embryonic development? Eur J Obstet Gynecol Reprod Biol. 2019;3:100045.

59. Plachot M, Belaisch-Allart J, Mayenga JM, Chouraqui A, Tesquier A, Serkine $\mathrm{AM}$, et al. [Oocyte and embryo quality in polycystic ovary syndrome] Qualite des ovocytes et embryons dans le syndrome des ovaires polykystiques. Gynecol Obstet Fertil. 2003;31(4):350-4.

60. Adolfsson E, Porath S, Andershed AN. External validation of a time-lapse model; a retrospective study comparing embryo evaluation using a morphokinetic model to standard morphology with live birth as endpoint. JBRA Assist Reprod. 2018;22(3):205-14.

61. Bellver J, Ayllon Y, Ferrando M, Melo M, Goyri E, Pellicer A, et al. Female obesity impairs in vitro fertilization outcome without affecting embryo quality. Fertil Steril. 2010;93(2):447-54. 
62. Shah DK, Missmer SA, Berry KF, Racowsky C, Ginsburg ES. Effect of obesity on oocyte and embryo quality in women undergoing in vitro fertilization. Obstet Gynecol. 2011;118(1):63-70.

63. Sermondade N, Huberlant S, Bourhis-Lefebvre V, Arbo E, Gallot V, Colombani $\mathrm{M}$, et al. Female obesity is negatively associated with live birth rate following IVF: a systematic review and meta-analysis. Hum Reprod Update. 2019;25(4):439-51.

64. Bellver J, Pellicer A, Garcia-Velasco JA, Ballesteros A, Remohi J, Meseguer M. Obesity reduces uterine receptivity: clinical experience from 9,587 first cycles of ovum donation with normal weight donors. Fertil Steril. 2013;100(4):1050-8.

65. Rhee JS, Saben JL, Mayer AL, Schulte MB, Asghar Z, Stephens C, et al. Diet-induced obesity impairs endometrial stromal cell decidualization: a potential role for impaired autophagy. Hum Reprod. 2016;31(6):1315-26.

66. Ujvari D, Jakson I, Babayeva S, Salamon D, Rethi B, Gidlof S, et al. Dysregulation of in vitro decidualization of human endometrial stromal cells by insulin via transcriptional inhibition of forkhead box protein O1. PLoS One. 2017;12(1):e0171004

67. Bellver J, Martinez-Conejero JA, Labarta E, Alama P, Melo MA, Remohi $J$, et al. Endometrial gene expression in the window of implantation is altered in obese women especially in association with polycystic ovary syndrome. Fertil Steril. 2011;95(7):2335-41.e1-8.
68. Glujovsky D, Farquhar C, Quinteiro Retamar AM, Alvarez Sedo CR, Blake D. Cleavage stage versus blastocyst stage embryo transfer in assisted reproductive technology. Cochrane Database Syst Rev. 2016(6):CD002118. https://doi.org/10.1002/14651858.CD002118.pub5.

69. Cedergren Ml. Maternal morbid obesity and the risk of adverse pregnancy outcome. Obstet Gynecol. 2004;103(2):219-24.

70. Steegers-Theunissen R, Hoek A, Groen H, Bos A, van den Dool G, Schoonenberg $\mathrm{M}$, et al. Pre-conception interventions for subfertile couples undergoing assisted reproductive technology treatment: modeling analysis. JMIR Mhealth Uhealth. 2020;8(11):e19570.

71. Oostingh EC, Koster MPH, van Dijk MR, Willemsen SP, Broekmans FJM, Hoek A, et al. First effective mHealth nutrition and lifestyle coaching program for subfertile couples undergoing in vitro fertilization treatment: a single-blinded multicenter randomized controlled trial. Fertil Steril. 2020;114(5):945-54.

\section{Publisher's Note}

Springer Nature remains neutral with regard to jurisdictional claims in published maps and institutional affiliations.
Ready to submit your research? Choose BMC and benefit from:

- fast, convenient online submission

- thorough peer review by experienced researchers in your field

- rapid publication on acceptance

- support for research data, including large and complex data types

- gold Open Access which fosters wider collaboration and increased citations

- maximum visibility for your research: over $100 \mathrm{M}$ website views per year

At BMC, research is always in progress.

Learn more biomedcentral.com/submissions 